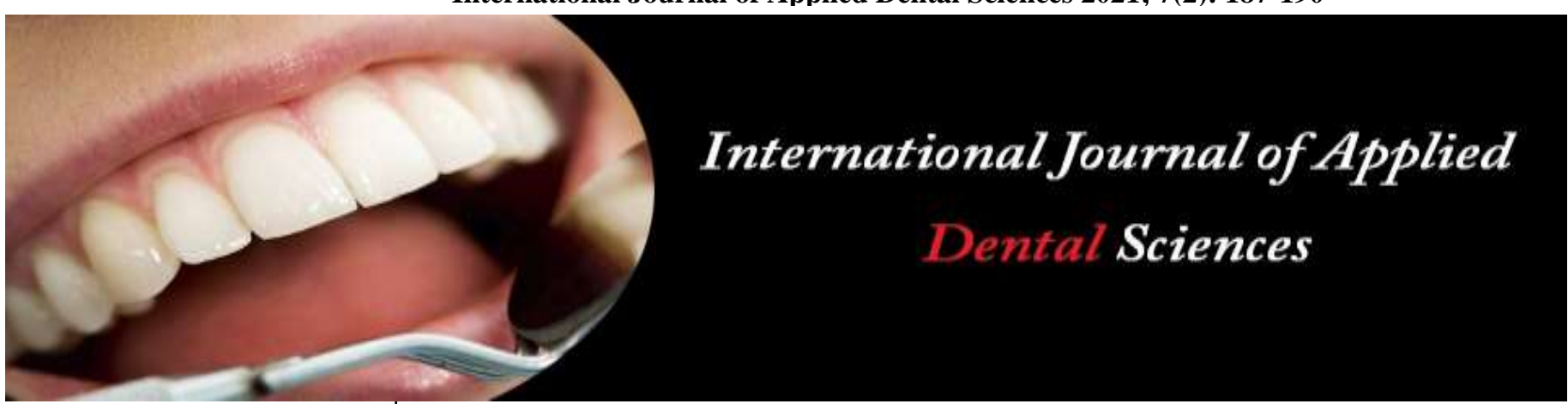

ISSN Print: 2394-7489

ISSN Online: 2394-7497

IJADS 2021; 7(2): 187-190

(C) 2021 IJADS

www.oraljournal.com

Received: 02-02-2021

Accepted: 13-03-2021

Dr. Aashna Bhardwaj

Ajeenkya D.Y. Patil University,

Pune, Maharashtra, India
Corresponding Author: Dr. Aashna Bhardwaj Ajeenkya D.Y. Patil University, Pune, Maharashtra, India

\title{
Patient satisfaction in rural area
}

\section{Dr. Aashna Bhardwaj}

DOI: https://doi.org/10.22271/oral.2021.v7.i2c.1209

Abstract

As the healthcare industry is moving towards a more patient oriented/focused sector, it is important for providers to understand the complete course of action that satisfies a patient in a rural area and how it affects the practice.

Objective: Patient satisfaction is a significant and widely used metric for assessing health-care quality. The purpose is to learn more about clinical outcomes, patient retention, and medical malpractice lawsuits, as well as how they impact the delivery of timely, reliable, and patient-centred health care.

Keywords: Patient satisfaction, empowerment, experience, population, health, survey

\section{Introduction}

What is patient satisfaction?

Patient satisfaction is a metric that measures how happy a patient is with the health care they got from their provider ${ }^{[1]}$.

Patient satisfaction refers to how pleased people are with their healthcare both in and out of the doctor's office. Patient satisfaction is a measure of care quality that provides clinicians with knowledge about different aspects of medicine, such as the effectiveness of their care and their level of empathy.

Although customer satisfaction is always crucial when offering any form of service, it has recently gained prominence in the healthcare industry. Patients are seeking more say in their healthcare as a result of patient-centred healthcare reform, and they require a certain quality of service from their providers ${ }^{[2]}$.

According to Vocera's 2016 Rise of the Chief Experience Officer study, 64 percent of healthcare professionals agree that patient satisfaction is just as critical as patient safety and clinical process changes in their organizations.

As the industry continues to shift in this patient-centred direction, few hospitals can afford to neglect this form of interaction, making it important for providers to consider the complexities of a good satisfaction strategy.

\section{Why is patient satisfaction important?}

Patient satisfaction is an important and widely used measure for evaluating health-care quality. Health outcomes, patient retention, and medical malpractice lawsuits are all affected by patient satisfaction. It has an effect on the delivery of high-quality health care in a timely, effective, and patient-centred manner ${ }^{[3]}$.

Simply put, patient satisfaction is a measurement of how well a patient is handled. The "how good" aspect refers to a patient's satisfaction with the treatment they got, not necessarily the standard of care.

Patient satisfaction is becoming highly critical in assessing the effectiveness of hospital treatment. The patient experience is becoming increasingly relevant in hospitals.

Over half $(54 \%)$ of healthcare executives say patient satisfaction is one of their top three priorities, according to Health Leaders Media's 2013 Industry Survey ${ }^{[2]}$.

How is patient satisfaction measured?

Although patient satisfaction can be assessed in a variety of ways, the most common is the 
Hospital Customer Evaluation of Healthcare Providers and Systems (HCAHPS) survey, according to studies. Via questions about patients' most recent hospital stay, this study, supported by the Centres for Medicare \& Medicaid Services, gleans insights into hospital quality and patient satisfaction.

Questions about patients' hospital experiences, questions to guide patients to relevant questions, questions to adjust for varying patient populations, and questions to answer Congress-mandated hospital quality reports are all included in HCAHPS surveys ${ }^{[2]}$.

\section{What factors affect patient satisfaction?}

Understanding what makes a patient work is on many providers' minds. Making patient satisfaction a priority in one's care delivery includes being able to assess what does and does not impact patient satisfaction.

When you realize that everyone is different, and we all have different priorities when it comes to our treatment, that job becomes immense.

Five factors that mainly affect patient's satisfaction:

- Expectations are a positive thing. Take a few moments at the beginning of the appointment to ask patients what they expect to get out of it.

- Communication is important. Boost your communication by being more upbeat.

- Take command. Give up some influence over the visit and practice making decisions together.

- Time has been invested. When the duration of the visit increases, so does the degree of satisfaction.

- Appearance

\section{How does technology help boost patient satisfaction?}

"I think it's very important to be able to continue the discussion outside of the workplace whether you're a patient at a primary care practice or you have any heart problems and you have an on-going relationship with a cardiologist, " Claim explains. "And once you've done that a couple of times, you start to feel a bond with your provider, and you get the feeling that they're invested in your health and well-being.

Improved health IT can also help increase hospital quality, which has an effect on patient satisfaction.

Technology often improves healthcare. This is true in both medical practice (robotic surgery improves outcomes) and healthcare delivery industry (analytic software helps improve quality). Many healthcare organizations, including rounding technology, are using technology and data ${ }^{[5]}$.

Despite the fact that patient satisfaction is a subjective measure, a healthcare organization must adopt a patient experience plan for many reasons:

- Patient satisfaction has become a competitive differentiator in a fragmented market as a result of the consumerization of healthcare.

- Patient satisfaction is commonly used as a measure for measuring the success of healthcare providers.

- The level of patient satisfaction is significant ${ }^{[5]}$.

"It's also about the personal touches."

Although health technology can help providers increase patient satisfaction, they must note that it all begins with them.

According to study, despite the technical standard of treatment given, provider empathy is the most significant indicator of whether or not a patient will be happy. Patients consider empathic care to be technically superior to less personalized care.
Also minor improvements, such as making more eye contact with patients, may increase satisfaction and commitment. According to a recent study published in the New England Journal of Medicine, 59 percent of patients said that spending more time in person with their doctors increased their commitment and satisfaction.

\section{Reality of healthcare in rural India}

Healthcare is a human right, but in India, 60 percent of the population lacks access to it due to a shortage of quality infrastructure, a scarcity of trained medical staff, and a lack of basic medicines and medical facilities. The bulk of the 700 million people live in rural areas, where medical services are in poor condition. Given the bleak picture painted by the truth, there is a pressing need. Despite the fact that the government operates numerous policies and initiatives, the success and usefulness of these programs is challenged due to inconsistencies in implementation. In rural India, where the number of primary health care centres (PHCs) is small, $8 \%$ of PHCs lack doctors or medical staff, 39\% lack lab technicians, and $18 \%$ lack a pharmacist.

In addition, India has the highest number of maternity deaths. The bulk of these occur in rural areas with inadequate maternal health services. And in the private sector, health care is often limited to family planning and antenatal care, with little provision for more essential facilities such as labour and childbirth, where adequate medical care can save lives if problems arise.

\section{The problems}

Due to non-accessibility to public health care and low quality of health care services, a majority of people in India turn to the local private health sector as their first choice of care. If we look at the health landscape of India 92 percent of health care visits are to private providers of which 70 percent is urban population. However, private health care is expensive, often unregulated and variable in quality. Besides being unreliable for the illiterate, it is also unaffordable by low income rural folks.

To control the spread of diseases and reduce the growing rates of mortality due to lack of adequate health facilities, special attention needs to be given to the health care in rural areas. The key challenges in the healthcare sector are low quality of care, poor accountability, lack of awareness, and limited access to facilities.

Various organizations are coming together for improvements in health care and technology plays a crucial role to facilitate this. Information and communications Technology provides hosts of solutions for successful implementation of these changes ${ }^{[6]}$.

\section{Technology for rural healthcare}

Technology has simplified many aspects of life for people who live in rural and remote areas. Applications that support instant communication and networking, as well as devices and machines that reduce reliance on city or urban-based facilities are some of the solutions that improve daily life. This has been a significant challenge in healthcare, especially for patients who need long-term care ${ }^{[9]}$.

Gram Vaani integrates cutting-edge mobile and IVR solutions in the field to simplify processes and apply best practices. For engaging with the difficult-to-reach customers at the bottom of the pyramid, they provide services to the healthcare industry, the social sector, and corporate organizations. 
They've created easy mobile applications to meet the needs of various industries and verticals. Thousands of lives in rural India have been affected as a result of our clients' enhanced systems and functions. They have a broad demographic scope across our mobile and IVR services. Our project aims to provide our partners with the best tools and strategies for reaching out to the rural market ${ }^{[6]}$.

\section{Research Methodology}

\section{Design}

Based on a literature review and data from the following group, we developed a questionnaire to assess patient experience and satisfaction with inpatient and outpatient care.

\section{Location}

Four dental care health facilities and clinics in the region of Uttar Pradesh and Haryana.

\section{Participants}

Number of patients: 100

i.e. 25 patients each clinic.

Aim

Patient evaluations of healthcare experiences

\section{Results}

Table 1: Shows in category yes and no

\begin{tabular}{|c|c|c|}
\hline Category & Yes & No \\
\hline Communication with assistant & $52 \%$ & $48 \%$ \\
\hline Communication with doctor & $94 \%$ & $6 \%$ \\
\hline Environment & $77 \%$ & $23 \%$ \\
\hline Medicine delivery & $77 \%$ & $23 \%$ \\
\hline
\end{tabular}

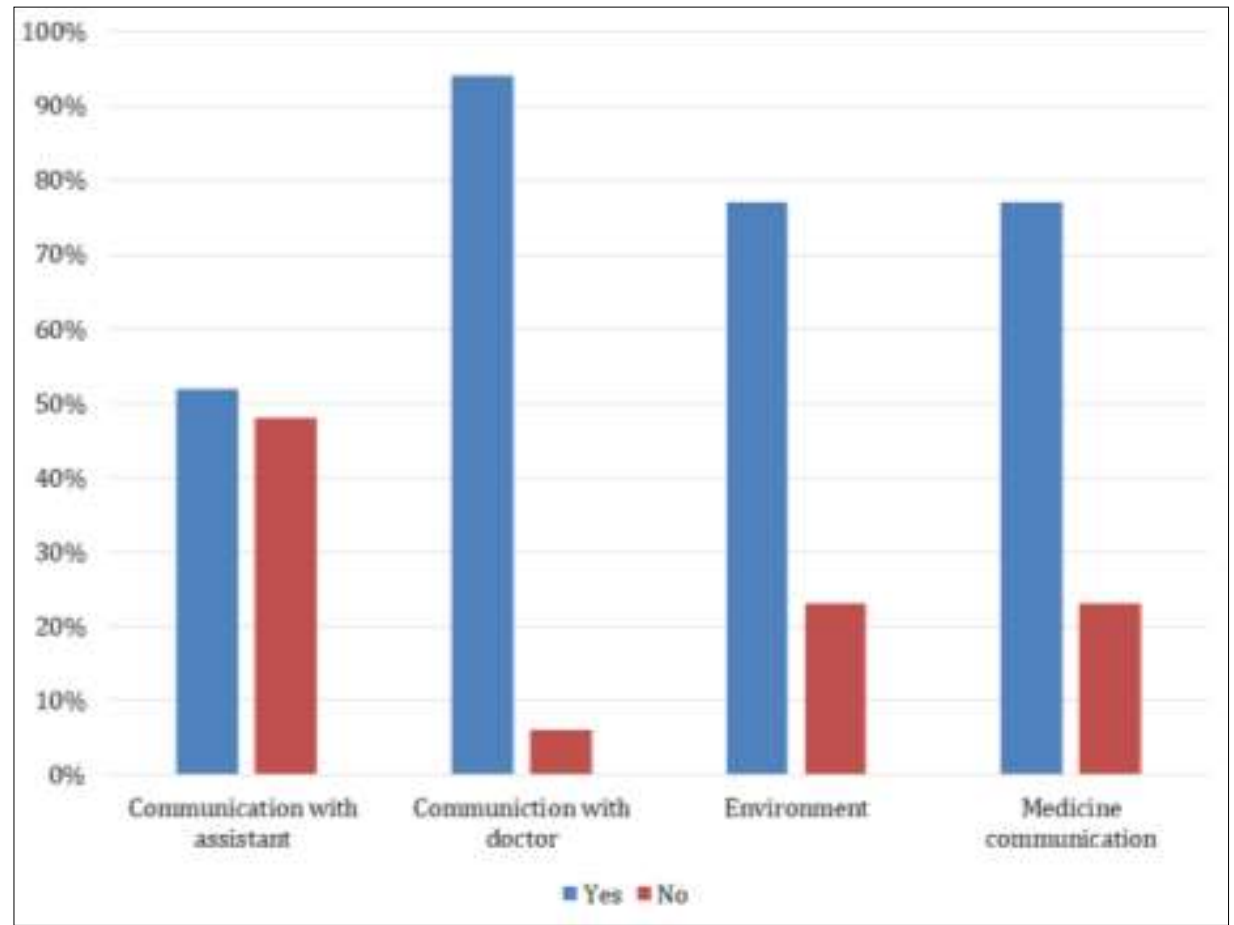

Fig 1: Chart Title

\section{Interpretation}

The above questionnaire was helpful in determining the level of satisfaction with healthcare delivery among low-income patients. +6 .

\section{Conclusion}

In general, the best way to increase patient satisfaction is to behave honestly and consistently. We as a healthcare professionals should always focus on the following:

- Address the patient by his/her name: The patient is more relaxed and connected. Wearing a nametag often makes it easy for patients to address you by your given name.

- Use of simple language: A doctor should devote time to patient education and deliver it in a language that the patient can comprehend.

- Patient friendly environment: There should be no noise or harsh lighting, it should be safe and sterile, and there should be no long wait times.

- Staff should be co-operative: A friendly, knowledgeable staff is vital to the success of a practice and the satisfaction of its patients.
The secret to a fully happy patient is a team that can foster a cooperative environment while providing the highest level of care and behaviour for patients in a timely manner ${ }^{[8]}$.

\section{References}

1. www.wikipedia. com

2. Patient Satisfaction and HCAHPS: What It Means for Providers.

https://patientengagementhit.com/features/patientsatisfaction-and-hcahps-what-it-means-for-providers

3. Bhanu Prakash. Wolters Kluwer - Medknow Publications, Patient Satisfaction. https://www. ncbi.nlm.nih.gov/books/NBK2682/

4. FPM EDITORS, Adapted from "What Do We Really Know About Patient Satisfaction." https://www. aafp. org/journals/fpm/blogs/inpractice/entry/improve_patient_ satisfaction. html

5. Ways Technology Improves Patient Experience in Healthcare, Sutherland. https://www. sutherlandglobal. com/our-thinking/5-ways- 
technology-improves-patient-experience-in-healthcare

6. Gramvaani, Rural Health Care: Towards a Healthy Rural India.

https://gramvaani. org/?p = 1629

7. https://academic.

com/inhqc/article/23/3/258/1794/153

oup.

8. Soben Peter. Essentials of public health dentistry (community dentistry) $5^{\text {th }}$ edition.

9. Tasneem Gould. Clinical Product Owner, OH Blog. How can technology benefit healthcare in rural communities? https://blog.orionhealth.com/how-can-technologybenefit-healthcare-in-rural-communities/ 